\title{
Physical Phenomena Observed during Strong Electric Discharges into Layered Y123 Superconducting Devices at $77 \mathrm{~K}$
}

\author{
Claude Poher (Corresponding author) \& Danielle Poher \\ Laboratoire Aurora - 33 Chemin de la Bourdette, F31400, Toulouse, France \\ Tel: 33-562-177-896 E-mail: claude.poher@wanadoo.fr
}

Received: May 4, 2011 Accepted: May 18, $2011 \quad$ Published: November 1, 2011

doi:10.5539/apr.v3n2p51

URL: http://dx.doi.org/10.5539/apr.v3n2p51

\begin{abstract}
Electric discharges of several megawatts were applied, at $77 \mathrm{~K}$, to propelling devices made of Y123 superconducting layers and thin insulating layers. During the discharges, the devices were strongly pushed in the direction opposed to the electron flow. The layered devices were apparently propelled by their emission of a momentum-bearing flux of an unknown nature. This flux weakly accelerated distant irradiated matter and created several physical effects not yet reported. The emitted beam had no electric charge, and traveled through materials without apparent absorption or dispersion, at a speed much greater than $1 \%$ the speed of light. The kinetic energy transferred by the propelling momentum of the devices to external masses, were proportional to the square of the electric energy of the discharges. No known effects were found which could explain these phenomena.
\end{abstract}

Keywords: Electric propulsion, Superconductors applications, Inertia, Gravitation, Dark matter, Dark energy, Energy generation

\section{Introduction}

The motivation for the present work originated from the experimental testing of an electric space propelling system we invented and patented (C. Poher, 1992; C. Poher, 2006). This device, called "emitter" uses thick layers of Y123 superconducting material and thin layers of insulating material that is a derivative of the Y123 material.

The propelling phenomenon we observed during these experiments seems to be tied with the inertia phenomenon acting upon very strongly accelerated electrons inside the device. However, the propelling emitter shows simultaneously an energetic behavior and diverse distant physical effects implying an interaction with an external source of energy. In order to satisfy energy conservation, we had effectively to suspect an interaction of the accelerated electrons with a field of an unknown nature surrounding the experimental apparatus.

In experiments reported here, a static superconducting material was used with internal free electrons strongly accelerated during electric discharges of short duration. Distant acceleration of irradiated matter and propulsion of the emitting material were observed. The second effect, the most conspicuous, has not been reported before.

\section{Experimental setup and methods}

\subsection{Principle of the experiments}

The experiments (Figs. 1 and 2) consisted in the application of high-voltage electric discharges (megawatts) of direct current lower than the critical current, generated from a bank of charged capacitors, into a patented (C. Poher, 2006) device made of superconducting material bathing in liquid nitrogen. The time course of the discharge current was related to the charge voltage of the energy storage capacitor $\mathrm{C}$ and to the values of the real elements of the circuit (Fig. 2). We chose and measured: $C=46.86 \mu \mathrm{F}, R \mathrm{c}=0.081 \Omega, R \mathrm{~s}=0.130 \Omega, L \mathrm{c}=0.80$ $\mu \mathrm{H}$. Our device, called "emitter" EM (see below) had a fixed internal resistance of the order of 0.01 to $0.05 \Omega$ that is proper to each emitter.

We observed physical effects of the discharge directed both upward, on the emitter support, and downward, away from the emitter, along the vertical axis $\Phi$. of the discharge current, with several detectors located far from the emitter (Fig. 3). Sets of measurements were made for different discharge voltages, with different 
emitters or with different kinds of metallic conductors replacing the emitter, inside liquid nitrogen or in the air. Experiments of the same type were done during the cooling down and during the warming up of the emitter, when the state of the S1 and S2 layers changed from conductive to superconductive and vice versa. We also observed discharges in other kinds of materials, such as piezoelectric and ferroelectric devices.

\subsection{Emitter}

The assigned role of the patented emitter was to accelerate strongly $\left(>10^{15} \mathrm{~m} / \mathrm{s}^{2}\right)$ numerous electrons $\left(>10^{21}\right)$ in the vertical up $->$ down direction, during short $\left(\approx 3.10^{-5} \mathrm{~s}\right)$ electric discharges, without distorting simultaneously, in the opposed direction, the lattice atoms of the material as this is the case inside resistive materials.

Emitters were made of two high temperature superconductive material thick films $(30 \mu \mathrm{m}) \mathrm{S} 1$ and S2 of the same chemical composition (Fig. 1) deposited on metallic electrodes. The useful acceleration of electrons occurred mainly inside the grains of S1 and S2 that were situated very close to insulated thin films $(<1 \mu \mathrm{m})$, made of a non conducting material existing between layers S1 and S2 and the metallic electrodes e+ and e-. Electric field was effectively the largest inside the insulated layers but did not stop abruptly at their frontiers. There was also an accelerating electric field between some grains of S1 and S2 where the electric contacts were not perfect.

Layers S1 and S2 had a larger superconducting critical temperature $(\approx 90 \mathrm{~K})$ than the boiling temperature of liquid nitrogen $(77 \mathrm{~K})$. The sintered material of layers $\mathrm{S} 1$ and $\mathrm{S} 2$ was a classical cuprate $\mathrm{YBa}_{2} \mathrm{Cu}_{3} \mathrm{O}_{7-\mathrm{x}}$ which method of fabrication has been described by many authors (M. K. Wu, 1987; B. Raveau, 1988; M. Murakami, 1992; U. Naoki, 1992). The insulated interface thin films were made of $\mathrm{YBa}_{2} \mathrm{Cu}_{3} \mathrm{O}_{5}$ material formed by local loss of oxygen atoms during the sintering process, this creating also conducting surface oxide on the metallic electrodes.

Our method of fabrication was classical: micron size grinded powders of $\mathrm{Y}_{2} \mathrm{O}_{3}, \mathrm{BaCO}_{3}, \mathrm{CuO}$ were mixed and heated up during 24 hours at $830^{\circ} \mathrm{C}$ under partial vacuum ( 2 to $30 \mathrm{hPa}$ ) and oxygen flow $(120 \mu \mathrm{g} / \mathrm{s})$. The S1 and S2 layers were sintered at $900{ }^{\circ} \mathrm{C}$, under the same partial vacuum. We made and tested more than one hundred such bi-layered emitters with an area of 9 to $50 \mathrm{~cm}^{2}$. These displayed a propelling performance proportional to the film area.

\subsection{Emitter support}

The emitter was bolted between two flat electrodes at the tip of a metallic support (Fig. 3). This support offered a low electrical resistance to the intense discharge current, and transmitted the momentum from the emitter to an insulated rod pushing the mobile magnets of a linear alternator. The emitter support was made of two bolted flat elastic L-shaped stainless steel bars, with a horizontal part forming a parallelogram attached to the setup, and a vertical part maintaining the emitter axis in position while permitting a slight vertical movement of a few mm only. Metallic bars had a $40 \pm 2 \mathrm{~mm}^{2}$ section. Because of this mechanical configuration, the emitter, its electrodes, and their support could not move independently.

\subsection{Discharge system}

The $46.86 \pm 0.01 \mu \mathrm{F}$ energy storage capacitor $\mathrm{C}$ (Fig. 1 and 2) was made of ten polypropylene $4.7 \mu \mathrm{F} \pm 5 \%$ capacitors, insulated to $7500 \mathrm{~V}$, and connected in parallel. It could be charged up to $4000 \mathrm{~V}$ by an external DC power supply. Discharges of up to 10,000 A were done through a large thyristor, insulated to $4500 \mathrm{~V}$, and capable to withstand a 13,000 A surge current.

\subsection{Emitter momentum sensor, the linear alternator}

The momentum from the emitter was carried up by the metallic support and transferred by contact to an insulated rod suspended by flat springs. The rod transferred itself the momentum to the moving magnets of the linear alternator, magnets also suspended by flat springs. The stator of that alternator was bolted to a separate support (Fig. 3). The momentum transfer stopped the movement of the emitter support. So this one remained macroscopically almost at rest. In order to obtain an efficient momentum transfer, the total moving masses of the rod and of the mobile magnets were chosen equal to the total moving masses of the emitter plus its support.

During a discharge, the alternator windings delivered a voltage proportional to the velocity of its mobile magnets, so the alternator output peak voltage was proportional to the transmitted momentum. An electronic circuit with a digital voltmeter measured this peak voltage. We calibrated this momentum sensor experimentally $(9.71 \pm 2 \% \mathrm{~g} . \mathrm{m} / \mathrm{s}$ per peak Volt without connected charge). 


\subsection{Detectors in the double Faraday's cage}

Distant detectors were shielded from electromagnetic fields by a double Faraday's cage made of two $0.8 \mathrm{~mm}$-thick aluminum enclosures. This cage was well insulated from the vibrations of the emitter system by independent supports. Inside the one meter height cage, eight drawers were put up for detectors. The bottom of the upper drawer was $26 \pm 0.5 \mathrm{~cm}$ under the emitter level. Four kinds of detectors performed measurements far from the emitter, along its vertical axis $\Phi$ (Fig. 3). (i) Several accelerometers with piezoelectric and inductive sensors, delivering voltages proportional to the acceleration or to the speed of tiny non-ferromagnetic masses placed along the axis $\Phi$.(ii) Flat capacitors with electrodes perpendicular to $\Phi$. (iii) Electric conductors aligned with $\Phi$. These three kinds of sensors were compensated for the residual electromagnetic field existing inside the Faraday's cage during discharges. (iv) A flat tank of water the surface waves of which were recorded by light reflection at grazing incidence by a video camera. For the sake of brevity the measurements from all these detectors will not be described, except those from the piezoelectric accelerometer, as it helped showing the existence of an emitted propelling flux and measuring one of its characteristics.

\subsubsection{Piezoelectric sensor accelerometer}

We used an industrial stack of several hundred piezoelectric elements made of PZT material, and connected in parallel. This sensor was an actuator P888-91 from PI, a German industry. The proper mass of the piezoelectric elements acted as an accelerometer mass, pushed by the emitted flux from the emitter. This stack was placed vertically along the axis of the emitter. This detector could be moved around, in order to determine the intensity distribution of the propelling flux along the propagation axis or at some distance from it (29 to $95 \mathrm{~cm}$ away). This piezoelectric sensor was able to measure a force less than $30 \mu \mathrm{s}$ in duration and more than $0.02 \mathrm{mN}$ in intensity. The detector was calibrated by the impact of a tiny body of known mass falling from several known heights. By adding known masses of different not ferromagnetic materials on top of the stack we were able to adjust the sensitivity of this accelerometer, and check that the force was proportional to the total irradiated mass, as this should be the case for an acceleration.

\subsection{Data recording}

Mechanical phenomena evolving slowly were recorded by a distant video camera at rate $25 \pm 0.01 \mathrm{frames} / \mathrm{s}$, and electric phenomena evolving rapidly were recorded by a digital memory oscilloscope (256 levels or $\pm 0.4 \%$ of full scale), enclosed in an independent double Faraday's cage, and triggered through an optoelectronic circuit.

The time window of the oscilloscope was chosen short enough (from -30 to $+70 \mu \mathrm{s}$ ) for the aerial sounds and mechanical vibrations, caused by the discharge, to travel less than $2 \mathrm{~cm}$ during this time, and so to avoid their recording by the sensitive sensors inside the Faraday's cage.

\subsection{Taking care of the electromagnetic effects on the Faraday's cage sensors}

The intense and brief discharge current into the emitter created a strong electromagnetic field, not completely eliminated by the double Faraday's cage. A voltage was induced in some of the sensors by the residual electromagnetic field, and this had to be taken into account in order to detect the signal induced by the phenomenon under study. As shown below, the voltage induced in the detectors by the electromagnetic field was proportional to the discharge voltage, while the signal induced by the phenomenon under study was proportional to the square of the discharge voltage. So they could be discriminated by varying the discharge voltage as follows. First, the detectors output signals were recorded during several discharges into a layered emitter bathing in liquid nitrogen. Next, they were recorded with the same current discharged into a stack of copper plates (control) replacing the layered emitter, and creating only the same electromagnetic field.

\subsection{Experimental protocol}

Physical phenomena occurring during the discharges being quite brief, the displays of the digital instruments, the movement of the momentum transfer rod and the screen of the memory oscilloscope were recorded by a digital video camera for subsequent analysis on a computer. The following protocol was systematically used:

(1) The tested emitter was bolted on its support and the support bolted on the experimental setup. The cryostat was filled with liquid nitrogen and the liquid level completed from time to time to compensate for evaporation. Forty minutes without any action were needed to reach thermal equilibrium. Then the video camera was started for permanent recording of the instruments during phases 2-4.

(2) The storage energy capacitor $\mathrm{C}$ was charged up to $600 \mathrm{~V}$ by the external power supply. The digital memory oscilloscope was put in waiting mode, to be triggered by the thyristor command circuit. The automatic command circuit of the thyristor was started. Experimenters went away for safety, and for preventing induced vibrations. 
Within 10 seconds, vibrations of the experimental system were damped and ceased completely. At the end of a 10 -s waiting period, the thyristor and the oscilloscope were automatically triggered. After the discharge a recording of the oscilloscope screen image was manually made on an USB memory chip for ulterior recovery and use.

(3) All steps in sequence (2) were repeated with a $200 \mathrm{~V}$ increase of the charge voltage of C.

(4) After a charge voltage of $3600 \mathrm{~V}$ was reached, the experiment was repeated once again starting at $600 \mathrm{~V}$.

\section{Experimental results}

More than 3500 discharges were recorded and analyzed from April 2007 to March 2011, with 130 different emitters. For examples, see the video movie in reference (C. Poher \& D. Poher 2010 a) and Fig 4.

\subsection{Experimental conditions}

Typical emitter voltage and current waveforms during a discharge (Fig. 5) show that the emitter was only resistive, and the total duration of the discharge $(30 \pm 5 \mu \mathrm{s})$ was almost the same for each emitter. The time course of the instantaneous electric power applied to this emitter (Fig. 6) shows that the total energy transferred to the emitter was 3 to $4 \%$ of the stored energy. The peak current was equal to the charge voltage divided by 0.44 ohms.

The time width of the discharges, at half the peak power, was $12 \pm 5 \mu \mathrm{s}$, and $90 \%$ of the stored electric energy was discharged from 6 to $22 \mu \mathrm{s}$ after the onset of the discharge. The peak power of discharges was generally observed $10 \pm 2 \mu \mathrm{s}$ after their onset. The similarity of the discharge parameters resulted from the internal resistance $(R c+R s)$ of the discharge circuit, which was much larger than the emitter resistance Rem, and imposed by the non oscillating current constraint $\left[(R c+R s+R e m) \geq 2(L c / C)^{1 / 2}\right]$.

Four percent of the energy stored into the capacitor bank was dissipated in the emitter as heat. Most of the stored energy (95\%) was dissipated as heat by the other components of the discharge circuit, including the amortization resistor of $0.13 \Omega$. A small part of the stored energy $(<1 \%)$ was radiated as electromagnetic field.

\subsection{Effects observed during discharges}

\subsubsection{Propulsive momentum}

The main physical effect observed was an upward propulsive momentum of the emitter, in the opposite direction to the flow of electrons. During all discharges in the layered emitter larger than $800 \mathrm{~V}$, the mobile magnets of the linear alternator always jumped up. For example, during a $2863 \pm 1 \mathrm{~V}$ discharge in emitter EM8, the propulsive momentum was $125 \pm 5 \mathrm{gm} / \mathrm{s}$. In this example, the discharge duration being $16 \mu \mathrm{s}$, the corresponding average propulsive force was $7800 \pm 375 \mathrm{~N}$, and the average acceleration of the emitter support was $15,600 \pm 750 \mathrm{~m} / \mathrm{s}^{2}$.

Fig. 7 shows that, for a typical emitter, the momentum $P$ created into the emitter, and transferred to the mobile magnets of the linear alternator, was proportional to the electric energy of the discharge. Experimentally, for emitter EM2, $P=1.12 \times 10^{-8} U^{2}$ where $U$ is the charge voltage, the factor $1.12 \times 10^{-8}$ being expressed in $\mathrm{kg} . \mathrm{m} \cdot \mathrm{s}^{-1} \cdot \mathrm{V}^{-2}$. The electric energy $E_{\mathrm{e}}$ of the discharge is $E_{\mathrm{e}}=C U^{2} / 2$. So, with the storage capacitor $C=46.86 \mu \mathrm{F}$, $P=4.78 \times 10^{-4} E_{\mathrm{e}}$, the factor $4.78 \times 10^{-4}$ being expressed in $\mathrm{m}^{-1} . \mathrm{s}$.

Similar results were obtained with all emitters, showing that the propelling momentum was proportional to the product of the number of accelerated electrons by their acceleration inside the emitter. The variability between performances of different emitters appeared to be proportional to the films area.

When the emitter was turned upside down (reversed current direction) the momentum did not change and its direction remained opposite to the electrons flow.

\subsubsection{Effect on the distant accelerometer}

During discharges, several lower amplitude physical effects were observed inside the double Faraday's cage at a distance from the emitter, along the $\Phi$ axis of the discharge current, especially with the accelerometer. A typical example of the output signal amplitude of the piezoelectric accelerometer as a function of the discharge energy is shown in Fig. 8. This accelerometer was negligibly affected by the residual electromagnetic field. During discharges applied to a stack of copper plates (false emitter) creating the same electromagnetic field, it showed no output signal, only electronic noise. During experiments of Fig. 8, at a discharge voltage of $2900 \pm 1$ $\mathrm{V}$, the sensitive mass of the piezoelectric accelerometer was accelerated to $0.125 \pm 10 \% \mathrm{~m} / \mathrm{s}^{2}$. This corresponds to a transferred momentum of $8.8 \times 10^{-8} \mathrm{~kg} \cdot \mathrm{m} / \mathrm{s}( \pm 10 \%)$. From the tests described in subsection 2-6, the force acting on the piezoelectric sensor was found to be proportional to the mass irradiated by the propelling flux (this is an acceleration) and to be independent of the nature of the irradiated mass of the accelerometer. 


\subsubsection{Other physical effects}

Three other physical effects were simultaneously observed during discharges into the emitters: emission of sound, emission of light and pushing of free electrons inside Faraday's cage sensors.

\subsubsection{Sound}

During all discharges larger than $500 \mathrm{~V}$, a particular loud sound was heard and recorded. This was a short $(<300$ $\mathrm{ms}$ ) and powerful whiplash-like sound, with an abrupt start $(<25 \mu \mathrm{s})$. It was recorded by the video camera at 44 $\mathrm{kHz}$ sampling rate (listen to it in video of reference (C. Poher \& D. Poher 2010 ar). No definite source was found for this sound apparently emitted by the whole experimental apparatus. Its intensity was correlated with the observed propulsive momentum. No such sound was heard or recorded during discharges applied to dummy emitters or devices other than the tested layered emitters.

\subsubsection{Light}

During all discharges larger than $1000 \mathrm{~V}$, a brief flash of light coming from inside the cryostat was noticed. This flash was recorded by the video camera, at least when the electronic shutter of its CCD sensor was not closed at the discharge instant. It was only visible on a single video image per discharge with unknown automatic individual exposure duration of less than $40 \mathrm{~ms}$. The light flash was too brief for obtaining an exploitable spectrum with our equipment. A rough RGB photometry of the emitted light was obtained from the color video image. The photometry ratios suggest, by comparison with nitrogen spectroscopic tables, a compatibility with nitrogen emission lines in visible light. The agitation of the boiling surface of liquid nitrogen did not allow exploitable pictures of the light source. The best we have been able to do was to use the shadows projected by the light on the front face of the instruments, to determine an approximate size and position of the light source inside the cryostat. Shadows geometry suggests the light source volume was small $\left(<5 \mathrm{~cm}^{3}\right)$, and located between the lower end of the emitter support and the bottom of the cryostat. No light was observed during discharges into a dummy emitter made of a stack of copper plates or devices other than the tested layered emitters. No light was observed during discharges in our layered emitters in the absence of emitter momentum, for example during the emitter cooling down.

\subsubsection{Pushing of electrons and of water molecules}

Appropriate detectors placed inside the Faraday's cage, such as a triple capacitor and a double solenoid, showed that the propelling flux pushed electrons inside dielectrics and conductors, and that it was not made of charged particles. The propelling flux created tiny waves on the surface of irradiated water. These effects will not be discussed here.

\subsection{These physical effects are specific to the tested layered emitters}

Three hundred discharges have been recorded in other materials than the tested layered emitters : (i) In normal conductors made of aluminum, brass or copper, both at room temperature and in liquid nitrogen (see the video movie (C. Poher \& D. Poher 2010 a) for a discharge applied to a stack of copper plates). (ii) In emitters of the same type of material, but of a different chemical composition. (iii) In fully superconductive emitters with no layers. (iv) In emitters where the materials composing the layers were mixed together before the final thermal treatment. (v) In piezoelectric and ferroelectric materials such as $\mathrm{BaSrTiO}_{3}, \mathrm{PZT}$, and PLZT, at room temperature, with a $300 \pm 10 \% \Omega$ resistance in parallel, allowing the discharge, as these materials are dielectrics. During discharges in all those materials, none of the effects described previously were observed.

These physical effects disappeared when the emitter layers were not superconducting. Discharges were applied to layered emitters at variable temperature. While the emitter was cooling down or warming up, respectively when liquid nitrogen was poured into the cryostat, and when it had evaporated, the progressive appearance and disappearance of the effects described previously was recorded. The full evolution took about 5 to 10 minutes. For stable effects, the emitter had to be at thermal equilibrium, at a temperature lower than the critical superconducting temperatures of the two layers. The present results were obtained with stable thermal equilibrium of the emitters during all discharges. This is the reason why 40 minutes waiting time were allowed in the experimental protocol.

\subsection{Properties of the emitted flux}

Properties of the flux were determined from the detectors located inside the Faraday's cage.

\subsubsection{The radiance diagram of the emitter}

It was determined with the piezoelectric accelerometer. This sensor measured the variation of the acceleration of irradiated matter, during constant discharge voltage $(2500 \pm 1 \mathrm{~V})$, at a variable horizontal distance from the 
vertical axis $\Phi$ of the emitter, along several azimuths. The area of the sensor was smaller than the one of the emitter. Its output voltages, at constant distance from the emitter $(490 \pm 1 \mathrm{~mm})$ and variable lateral distances from $\Phi$ (up to $60 \pm 1 \mathrm{~mm}$ ), gave a rough radiance diagram. Repeating these measurements at different azimuths (0 to $360^{\circ}$ by $30 \pm 3^{\circ}$ steps), we observed that the radiance diagram was symmetrical around $\Phi$. The output voltage of the accelerometer was almost constant $( \pm 5 \%)$ in a circular area about the size of the emitter vertical projection, the acceleration diminishing away from the axis. An attenuation of $10 \%$ was recorded at $2^{\circ} \pm 0.2^{\circ}$ from $\Phi, 20 \%$ at $4.9^{\circ} \pm 0.2^{\circ}, 50 \%$ at $5.8^{\circ} \pm 0.2^{\circ}$, and $67 \%$ at $7^{\circ} \pm 0.2^{\circ}$. The radiance diagram had a $11.6 \pm 0.4^{\circ}$ summit angle for a $6 \mathrm{~dB}$ attenuation. The propelling flux propagation was anisotropic, with an axis and a direction extending from the electron flow axis into the emitter.

\subsubsection{The minimum propagation speed of the flux}

It was determined from the output signals of two detectors, insensitive to the residual electromagnetic field of the discharge, placed inside the double Faraday's cage as far apart as possible, their vertical distance being $95 \pm 0.1$ $\mathrm{cm}$ along $\Phi$. They were recorded simultaneously at a scale of $10 \pm 0.01 \mu$ s per division on the horizontal axis. Figure 9 shows an example of such an experiment repeated many times with different detectors. These measurements showed no time difference, up to $\pm 0.3 \mu \mathrm{s}$, between the onsets of the voltage variations on both detectors. With a propagation of $0.95 \pm 0.001 \mathrm{~m}$ in less than $0.3 \pm 0.01 \mu \mathrm{s}$, the speed of the flux was greater than $3.17( \pm 0.01) \times 10^{6} \mathrm{~m} / \mathrm{s}$, i.e. larger than $1 \%$ of $c$.

\subsubsection{No absorption of the propelling flux by inserted matter was found}

We used sets of two successive discharges in the same emitter, at the same voltage $(2500 \pm 1 \mathrm{~V})$, while recording the output signal of the piezoelectric accelerometer and of other detectors in the Faraday's cage. Before the second twin discharge, various thick pieces of matter: steel, wood, stone, paraffin wax, granite, aluminum, water, brass etc, were inserted above the accelerometer and other detectors, in the upper drawer position. No difference in the recorded output signals, with and without insertion of matter, was found up to the accuracy of our measurements $( \pm 0.4 \%)$.

\subsection{Kinetic energy transferred to the linear alternator and energetic efficiency}

With more than sixty small area layered emitters (about $1 \mathrm{~cm}^{2}$ ), we observed the following average momentum $P$ versus the discharge voltage $U$ :

$$
P=1.12 \cdot 10^{-8} U^{2}
$$

However, with fifty $25-\mathrm{cm}^{2}$ thin-films emitters such as $77 \mathrm{YC} 25$, we observed, on average :

$$
P=2.4 \cdot 10^{-7} U^{2}
$$

Consider now expression (1-1) and a free mass $m$ lifted up to a height $h$ by the momentum $P$, as in the case of the linear alternator in the present experiments. The mass initial vertical speed $V$ is $V=P / m$. The height $h$ attained by the mass in the earth gravitational field of acceleration $g$, when using the plain emitters obeying expression (1-1), is:

$$
h=V^{2} / 2 \mathrm{~g}=P^{2} / 2 \mathrm{~g} \mathrm{~m}^{2}=1.25 \cdot 10^{-16} \mathrm{U}^{4} / 2 \mathrm{~g} \mathrm{~m}^{2}
$$

The kinetic energy $E_{\mathrm{m}}$ transferred to mass $m$ is therefore:

$$
E_{\mathrm{m}}=m V^{2} / 2=m g h=6.27 \cdot 10^{-17} U^{4} / m
$$

The kinetic energy displayed by the linear alternator resulting from the propelling effect in the emitter, was therefore proportional to the square of the electric energy of the discharge. Indeed, the electric energy $E_{\mathrm{e}}$ stored into the discharge capacitor $C$ is proportional to $U^{2}$ :

$$
E_{\mathrm{e}}=U^{2} C / 2
$$

Therefore, the energetic efficiency of the whole experiment $\quad \eta=E_{\mathrm{m}} / E_{\mathrm{e}}$ was:

$$
\eta=1.25 \cdot 10^{-16} U^{2 / m C}
$$

The factor $1.25 .10^{-16}$ appearing in (5) is not a dimensionless constant. It is the square of the experimentally determined factor appearing in (1-1). With a small $m C$ value, and by increasing the discharge voltage $U$ it appears possible to get a positive energy balance between the kinetic energy of the lifted mass $m$ and the discharge electric energy, under the condition that the critical current density into the emitter is not attained. The $\geq 100 \%$ energy efficiency condition should be given by :

$$
U \geq 8.93 \cdot 10^{7}(m C)^{1 / 2}
$$


Now consider the same calculation made from experimental result (1-2) with a larger area emitter. Expression (6) becomes now, for a larger than $100 \%$ energy efficiency:

$$
U \geq 4.17 \cdot 10^{6}(m C)^{1 / 2}
$$

3.5.1 Verification of the relationship between the experimental energetic efficiency and the discharge voltage with the linear alternator.

Experimental results (3) and (5), apparently violating energy conservation, we first suspected that the measurement of the momentum with a horizontal pendulum we first used was incorrect (See video C. Poher \& D. Poher 2010 a). So we checked the form of expression (3) by replacing the horizontal pendulum by the linear alternator rated to $100 \mathrm{~W}$. First, we made sure that it delivered an output voltage proportional to the velocity of its mobile magnets, and found that this was obtained with an energy efficiency of $92 \%$. This type of alternator was also found to exhibit an excellent linearity when measuring a pulsed momentum $( \pm 0.1 \%)$.

The linear alternator was a Qdrive 1S102M model, rated to 100 watts. It had mobile magnets with a mass $m$ of $432 \pm 1 \mathrm{~g}$. When briefly pushed by the emitter support, the mobile magnets suspended by flat steel springs, freely oscillated up and down. So the stator coil delivered an alternating voltage which peak amplitude was proportional to the vertical speed of the magnets. After a momentum transfer from the emitter support, this alternator delivered an amortized AC voltage, at about $50 \mathrm{~Hz}$, during almost half a second. The peak voltage of the first oscillation was proportional to the transferred momentum. Its calibration factor determined experimentally was $9.71 \pm 2 \%$ g.m/s per peak volt. Measured with this alternator, discharges into a bi-layered emitter yielded a mechanical kinetic energy proportional to the square of the electric energy stored into the capacitor C (see Fig. 10 as an example). That confirmed again the form of expression (3).

We could not check expressions (6) or (7) with the linear alternator and discharges into layered emitters because, with $m=0.432 \mathrm{~kg}$ and $C=46.86 \mu \mathrm{F}$, the energy efficiency would exceed $100 \%$ for $U>400 \mathrm{kV}$ and $\mathrm{U}>18.7 \mathrm{kV}$ respectively. These voltages cannot be attained with our present experimental system, which is limited to $4 \mathrm{kV}$ discharges. This is the reason why we are planning for the future to use a specially built linear alternator with $m$ $=40$ grams.

3.5.2 Verification of the relationship between the experimental energetic efficiency and the discharge voltage with a slightly imbalanced horizontal pendulum.

Before using the linear alternator we measured the propelling momentum with a large moment of inertia horizontal pendulum slightly unbalanced by a small tip mass $m$ after careful adjustment of the pendulum static balance. At rest, this tip remained in contact with the insulated top of the vertical emitter support, in order to receive the created momentum.

The diamond shape horizontal pendulum (see movie (C. Poher \& D. Poher 2010 a)) had a total mass of $794 \pm 1 \mathrm{~g}$ and a length of $588 \pm 0.5 \mathrm{~mm}$. It was made of an aluminum alloy and had lead masses $(M=320 \mathrm{~g})$ at its two arms extremities. One tip received the supplementary mass $(m=1.498 \pm 0.002 \mathrm{~g})$.

During a discharge, the pendulum larger mass end jumped up to a height $h$ proportional to the square of the total momentum transferred to it. The maximum height $h$ attained, and the corresponding momentum and potential energy, were determined from video images and calibrations. The horizontal pendulum had a large moment of inertia, so it rotated slowly enough to have its movement recorded accurately by the video camera at 25 images/s. Because of its flat shape and low velocity, its aerodynamic drag was minimal.

The pendulum had a potential energy calibration factor of $14.4 \pm 1 \mu \mathrm{J}$ per $\mathrm{mm}$ of jump height $h$. It reached vertical position at $3.9 \pm 0.1 \mathrm{~mJ}$. The calibrated momentum versus jump height was $7.3 \pm 0.2 \mathrm{~g} . \mathrm{m} / \mathrm{s} \mathrm{per} \mathrm{mm} \mathrm{m}^{1 / 2}$. Its moment of inertia was $2.90 .10^{-2} \pm 0.01 \times 10^{-2} \mathrm{~m}^{2} . \mathrm{kg}$. The largest value of the momentum directly measurable from the height $h$ of the pendulum, corresponding to a full rotation of the pendulum, was $125 \mathrm{~g} . \mathrm{m} / \mathrm{s}$. However, measurement of its initial angular velocity was possible with the video camera by comparing successive images, and its kinetic energy could then be deduced knowing its moment of inertia.

We first used this pendulum method to try checking expression (7). This expression shows that, with the values of $m$ of the horizontal pendulum and the same value of $C$ as before, the energy efficiency should exceed $100 \%$ for $U>1100 \mathrm{~V}$, which could apparently be checked with our present experimental system. Experiments with thin films emitters and the horizontal pendulum, up to $U>1100 \mathrm{~V}$, yielded effectively an increasing energy efficiency, confirming expression (5), and a value of the pendulum kinetic energy approaching progressively the input electric energy. We went very close to expression (7) limit and very close to the $100 \%$ energy efficiency with a sufficient accuracy to show that this series of expressions reflect a true behavior. 
However, during these verification experiments with the horizontal pendulum, we could not measure accurately enough the kinetic energy with discharges voltages over $1000 \mathrm{~V}$ because the rotating movement of the pendulum became so violent that the pendulum end left the emitter support before full transfer of the emitter momentum.

Consequently, the emitter and its support began to jump up macroscopically, ejecting some of the liquid nitrogen out of the cryostat and condensing the surrounding atmospheric water vapor as fog, masking partially the pendulum movement to the camera. Even the heavy $(1.5 \mathrm{~kg})$ cryostat itself jumped up, because of the residual movement of the emitter support inside liquid nitrogen. Video (C. Poher \& D. Poher 2010 a) shows the same violent effects.

Increasing further the input electric energy being potentially dangerous and quite difficult to interpret, the discharge voltage was limited to $\sim 1150 \mathrm{~V}$ with the horizontal pendulum only method.

The experimental system should have to be considerably modified to continue this kind of verification, with a sufficiently low mass detector, able to accept the huge momentum created by the emitter.

The actual moving magnets linear alternator and the horizontal pendulum methods have shown here their own limits. We are now imagining the measuring means able to capture the huge created momentum and to attain the required level of accuracy.

Nevertheless we consider that this is only a technical question that will be solved in the future. So, the series of relations (1-1) to (7) have been experimentally confirmed by so many results that we should consider them to be a correct representation of the involved phenomena, with a quasi certainty.

\section{Discussion}

\subsection{Comments about the observed physical effects}

When powerful electric currents were discharged at $77 \mathrm{~K}$ in superconducting emitters, four physical effects were observed: propulsive momentum, distant acceleration of matter, emitted sound and emitted light inside the cryostat. They can be tentatively interpreted as resulting from the emission by the emitters of a propelling flux propagating at high speed. Flux apparently not absorbed by intercalated materials.

\subsubsection{Propulsive effect}

Due to the mechanical configuration of the setup, only a vertical movement of the whole emitter, pushing up its metallic support, could cause this effect. The propulsive momentum was produced by the discharge current inside the layered emitter, because it was not observed when replacing the emitter by other materials. And also because the propelling momentum amplitude increased with the discharge current, disappeared with it. The propulsive momentum remained the same when the emitter was turned upside down, its direction being always opposed to the direction of the electron flow. This momentum, created without ejection of matter, suggests the emission by the emitter of a downward invisible flux bearing this momentum. This "propelling flux" should be detected along its propagation path, towards the nadir, under the emitter.

\subsubsection{Distant acceleration of matter}

The propelling flux momentum was proportional to the energy of the discharge, (Fig. 7). So if this flux was able to transfer a part of its momentum to matter inserted along its path, the transferred momentum should also be proportional to the energy of the discharge and this was actually observed (Fig. 8). Because of momentum conservation, the flux was expected to bear the whole momentum of the emitter. However, the flux transferred only a small part $\left(\approx 10^{-6}\right.$ per gram of irradiated matter $)$ of its own momentum to the piezoelectric accelerometer mass. This fact was not caused by absorption of the propelling flux by the Faraday's cage, as shown in section 3.4. This indicates a very weak interaction cross section of the propelling flux with irradiated matter. Our results show that the propelling flux weakly accelerated matter, as the force was proportional to the mass of the irradiated matter. The flux pushed matter inserted along its propagation path. Several authors (E. Podkletvov, R. Nieminen, 1992; E. Podkletnov, G. Modanese, 2003; M. Tajmar, C. J. de Matos, 2003 \& 2005; M. Tajmar M., F. Plecescu, K. Marhold, C. J. de Matos, 2006) observed also fortuitously this distant phenomenon with the same properties.

\subsubsection{Sound emission}

A low time resolution oscillogram of that sound revealed an abrupt front $(<20 \mu \mathrm{s})$ suggesting a supersonic shock wave. This sound wave might have been emitted by the whole experimental apparatus, located under the emitter level, when irradiated by the propelling flux. This could explain why no definite source of this sound was found. 


\subsubsection{Light emission inside the cryostat}

The voltage applied to the emitter was only a fraction of the discharge voltage. During $2200 \pm 1 \mathrm{~V}$ discharges, only $\sim 400 \pm 40 \mathrm{~V}$ were applied across the emitter. This was not sufficient to ionize liquid nitrogen. The emission of light inside the cryostat could result from the excitation of nitrogen atoms by the electromagnetic radiation emitted by the accelerated electrons (Bremsstrahlung effect). However it is unlikely that this low energy radiation $(<3 \mathrm{keV})$ could have traveled through the dense emitter material and the electrodes before reaching nitrogen atoms. So this light emission resulted more likely from the propelling flux itself, since it was not absorbed by the inserted matter. The ionization would result from the pushing action of the flux on electrons of nitrogen atoms. No light emission was observed in the air but the atomic density is about 700 times larger in liquid nitrogen than in the atmosphere. The fact that no flash of light was observed during emitter cooling down, while there was not yet any emitter momentum, supports the hypothesis of an effect caused by the propelling flux. For this hypothesis to be correct, the interaction of the propelling flux with nitrogen atoms should transfer a kinetic energy to the electrons of the ionized nitrogen atoms greater than several electrons-volts.

4.1.5 Minimum propagation speed of the propelling flux

The twin-signal experiments showed that the propelling flux propagates at a velocity greater than $1 \%$ of $c$. Many repetitions of this experiment with different pairs of detectors confirmed this minimum propagation speed. If the propelling flux would propagate at the speed of light, the time difference between two events inside the Faraday's cage would be $\sim 3.3$ nanoseconds, a time interval too short to be measurable with our detectors and oscilloscope.

\subsubsection{Absence of absorption by inserted matter}

No noticeable absorption of the propelling flux by matter was found with any of the different samples we tested. The thickness, mass and density of the inserted matter had no observable effect, up to the $\pm 0.4 \%$ accuracy of our measurements. However, the propelling flux interacted weakly with the inserted matter, as shown by the piezoelectric accelerometer signal, and by the surface waves shown with the water tank. We should remark that gravitation is the only known interaction which is insensitive to the insertion of matter along its path and which interacts weakly with matter.

\subsection{Analysis of possible artefacts}

\subsubsection{Artefacts concerning the propelling momentum of the emitter}

Six possible artefactual sources for the emitter momentum were examined:

\subsubsection{Piezoelectric effect}

A reverse piezoelectric effect creates a tiny movement of the opposed sides of a piezoelectric crystal to which a voltage is applied. However, if a piezoelectric force would be present, the relationship between momentum and applied voltage would be linear and not quadratic as observed (Fig. 7). Moreover, a piezoelectric material should be a dielectric, whereas the tested emitters were conductive, almost short circuits (their resistance was lower than $0.05 \mathrm{ohms})$.

\subsubsection{Electromagnetic effect}

An electromagnetic field creates strong forces on currents in conductors and on ferromagnetic materials such as iron. However, electromagnetic forces are proportional to the current generating the field, not to the square of the current; no ferromagnetic materials were used; and no propulsive effect was observed when the emitter was replaced by a metallic conductor, of the same dimensions, submitted to the same current discharges.

\subsubsection{Interaction with the Earth magnetic field}

The interaction between the discharge current and the Earth magnetic field can create a force. However, the metallic bars configuration would create a small torque, instead of a propelling force greater than $80,000 \mathrm{~N}$ (largest peak force observed). Again, the force should be proportional to the current intensity, not to its square.

\subsubsection{Thermal effect}

A strong thermal effect inside liquid nitrogen could create an intense evaporation, and the nitrogen vapor could push the emitter support out of the cryostat. However, the discharge duration being extremely short $(30 \mu \mathrm{s})$, the vapor could not move sufficiently up to create the observed effect in synchrony with the discharge current. Moreover, the electric energy transferred to the emitter is small, only a few $\%$ of the stored energy $(<100 \mathrm{~J})$. So, the amount of liquid nitrogen that would change phase with the available thermal energy (less than $3 \mathrm{~J}$ ) would be insufficient to account for the observed effects, as liquid nitrogen needs $160 \mathrm{~J} / \mathrm{cm}^{3}$ for evaporation. Note also that 
during intense discharges, the heavy $(1.5 \mathrm{~kg})$ cryostat jumped up, which is unlikely from a thermal effect. Finally, if a thermal effect would be the cause of the propelling momentum, this momentum should be proportional to the square root of the discharge energy, this is not what is observed (Fig 7).

\subsubsection{Solid-state physics effects inside the emitter material}

Control experiments done with fully superconducting emitters of the same composition (but with no layers) did not show the effects found with our specific layered emitters. No effects were observed with discharges applied to emitters made of only a conductive layer type of material. Effects internal to the emitter cannot account for the acceleration of distant matter inside the Faraday's cage.

\subsubsection{Effect resulting from the electric field applied to liquid nitrogen}

When the coolant was poured into the cryostat, the propelling effect appeared after at least 10 minutes and then increased progressively, during each discharge, until the emitter temperature became uniform on the whole area of the layers. The full evolution required about 30 minutes. When liquid nitrogen had evaporated, the propelling effect diminished also progressively during 10 to 15 minutes while the temperature inside the emitter was rising above the critical superconducting temperature. The thermal inertia of the emitter, its electrodes and its stainless steel support was clearly involved.

\subsubsection{Artefacts about the propelling flux effects inside the Faraday's cage}

Three possible artefactual effects on the Faraday's cage sensors were also examined:

\subsubsection{Electromagnetic effect}

A strong electromagnetic field was radiated by the discharge current around the discharge circuit and induced currents in surrounding conductors as well as electric fields in surrounding dielectrics. According to Maxwell's equations, these inductions should be proportional to the derivative of the discharge current. The effects of this electromagnetic field were limited by using a double Faraday's cage. The cage sensors were designed to be almost insensitive to the residual electromagnetic field. Nevertheless, weak electromagnetic field effects were recorded. However, by comparing results of discharges applied to our emitters and to metallic conductors, two effects were clearly distinguished, one (small) arising from the electromagnetic field and the other, (strong) proportional to the square of the discharge current. The second effect disappeared completely when discharges creating the same electromagnetic field were applied to a metallic conductor instead of the layered emitters.

\subsubsection{Effect of a flux of known particles}

A flux of known elementary particles (such as electrons or protons) emitted by the emitter could push matter and induce currents or electric fields. However, the energy of these particles should be smaller than $3 \mathrm{keV}$ in all experiments reported here. Particles with a so small kinetic energy could not travel through materials placed along their path. Moreover, usage of flat capacitors showed that the hypothetic propelling flux could not be made of charged particles, and relatively small electric discharges in matter are not known to trigger the emission of neutrons.

\subsubsection{Pushing effect from a continuous spectrum of electromagnetic radiation}

A radiation caused by the Bremsstrahlung emission from accelerated and decelerated electrons was supposed in this analysis. However, the photons of this emission could not have an energy greater than that of the accelerated electrons $(<3 \mathrm{keV})$ so they could not travel through the interposed materials of the cryostat $(1 \mathrm{~mm}$ thick stainless steel), Faraday's cages $(2 \times 0.8 \mathrm{~mm}$ thick aluminum), plus several wood supports $(15-20 \mathrm{~mm}$ thick).

\subsection{Does the emitter interact with an external energy source?}

The discharge circuit appears as a "closed system" that should not generate any external momentum. However, the reported experiments showed a huge momentum transfer and the emitter support was submitted to accelerations reaching up to ten thousands $g$ 's. The effects observed are consistent with the emission by the emitter of an anisotropic propelling beam accelerating distant matter. It would explain why the emitter was pushed up, and the propelling flux should bear all the propelling momentum of the emitter. This raises the question of the origin of the energy carried by the flux. The source of energy was likely neither the emitter, because it was not progressively destroyed, nor the electric energy stored into the capacitors, because of the close to $100 \%$ efficiency found in the experiments reported in section 3.5 (expressions 3 and 5). The energy could come from an external source, for example the liquid nitrogen, but this is not consistent with the persistence of the propelling effect a few minutes after complete evaporation of nitrogen. Therefore, we suggest that an unknown interaction was triggered through the accelerated electrons inside the emitter and that the experimental 
setup was an open system interacting with an external energy source. The nature of this interaction can be studied (C. Poher, $2010 \mathrm{~b}$ ) from the experimental properties of the propelling flux as summarized now.

\section{Conclusion}

Main experimental properties of the propelling flux and consequences:

The flux bears a momentum proportional to the energy of the electric discharge transferred to the emitter.

It propagates in the direction of the electron acceleration inside the emitter.

The flux momentum seems to be proportional to the electron acceleration inside the emitter.

The flux is neither absorbed nor scattered by matter at rest, however it does weakly accelerate matter placed along its path (it pushes matter).

A small fraction $\left(\approx 10^{-6}\right.$ per gram $)$ of the momentum borne by the flux is transferred to irradiated matter.

The fraction of momentum transferred by the flux is proportional to the mass of irradiated matter.

The propagation speed of the flux is not known, it is much greater than $1 \%$ of $c$.

The flux is not made of charged particles.

It creates a sort of supersonic shock wave, when propagating through materials.

It apparently pushes electrons in dielectrics and in conductors.

It apparently pushes water molecules, generating waves.

It apparently ionizes liquid nitrogen if sufficiently intense.

These results offer the possibility to propel strongly a vehicle by applying electric discharges to a superconducting emitter without ejecting matter in space, and using only electric energy.

They also suggest the possibility of energy extraction from an unknown surrounding source, thus raising questions about the nature and properties of this source.

\section{References}

de Matos C.J., Tajmar M. (2005). Gravitomagnetic London moment and the graviton mass inside a superconductor. Physica C, 432, 167, http://dx.doi.org/10.1016/j.physc.2005.08.004

Murakami M. (1992). Processing of bulk YBaCuO. Supercond. Sci. Technol. 5, 185. http://dx.doi.org/10.1088/0953-2048/5/4/001

Naoki U. et al. (1992). Furukawa El. Co. Ltd., Method of manufacturing oxide superconductor and the precursor of the oxide superconductor. US Patent 5106824, April 21, 1992. [Online] Available: http://www.wikipatents.com/US-Patent-5106824/method-of-manufacturing-oxide-superconductor-and-the-precu rsor-of-the

Podkletnov E., Modanese G. (2003). Investigation of High Voltage Discharges in Low Pressure Gases Through Large Ceramic Superconducting Electrodes. Journal of Low Temperature Physics, 132, 239. http://dx.doi.org/10.1023/A:1024413718251

Podkletnov E., Nieminen R. (1992). A possibility of gravitational force shielding by bulk $\mathrm{YBa}_{2} \mathrm{Cu}_{3} \mathrm{O}_{7-\mathrm{x}}$ superconductor. Physica C, 203, 441, http://dx.doi.org/10.1016/0921-4534(92)90055-H

Poher C. (1992). Universons, a possible key to interstellar exploration. 43rd IAF, International Astronautical Congress, Washington DC. [Online] Available: http://adsabs.harvard.edu/abs/1992wadc.iafcW....P

Poher C. (2006). Device for propelling particles by means of acceleration and applications thereof. Europ. Patent pub. WO 2007/093 699 A2. PCT FR 2007/000249 Feb 14, 2006. [Online] Available: http://www.wipo.int/pctdb/en/wo.jsp?WO=2007093699

Poher C. $(2010 \quad$ b). Theoretical hypotheses. [Online] Available: http://www.universons.org/site_publication/Textes_Publication/Suppl_material_2.pdf

Poher C., Poher D. $(2010$ a). Experimental video movie clips. [Online] Available: http://www.universons.org/site_publication/Emitter_Momentum.mov

Raveau B. (1988). C.G.E., A method of manufacturing a superconductive conductor containing a ceramics superconductive material being in composition generally expressed by a formula AaBbCc. European Patent EP 0286823, October 19, 1988. [Online] Available: http://patent.ipexl.com/EP/EP0283024.html 
Tajmar M., de Matos C.J. (2005). Extended analysis of gravitomagnetic fields in rotating superconductors and superfluids. Physica C, 420, 56. http://dx.doi.org/10.1016/j.physc.2005.01.008

Tajmar M., Plecescu F., Marhold K., de MatosC.J. (2006). Experimental Detection of the Gravitomagnetic London Moment. ArXiv gr-qc/060303. [Online] Available: http://arxiv.org/abs/gr-qc/0603033

Tajmar M., de Matos C.J. (2003). Gravitomagnetic field of a rotating superconductor and of a rotating superfluid. Physica C, 385, 551. http://dx.doi.org/10.1016/S0921-4534(02)02305-5

Wu M. K., (1987). Superconductivity at $93 \mathrm{~K}$ in a new mixed-phase Y-Ba-Cu-O compound system at ambient pressure. Phys. Rev. Lett, 58, 908. http://dx.doi.org/10.1103/PhysRevLett.58.908

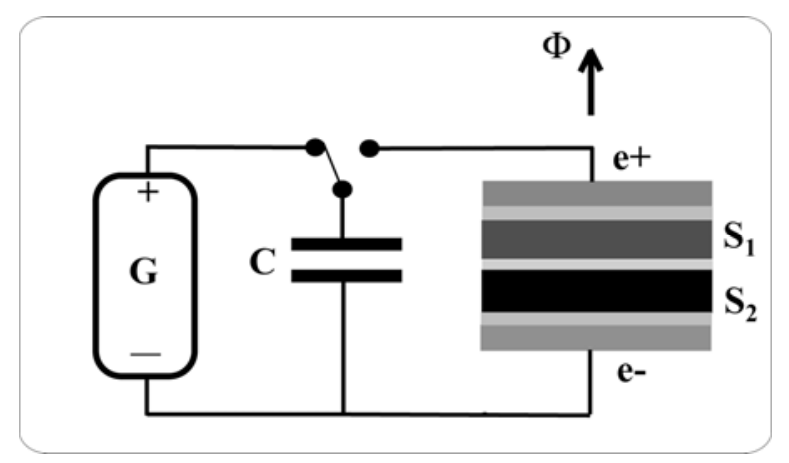

Figure 1. Principle of the experiment

An energy storage capacitor $\mathrm{C}$ is charged by a DC generator $\mathrm{G}$ to a voltage chosen between 0 and $4000 \mathrm{~V}$. Then a thyristor electronic switch connects the capacitor to the layered superconducting emitter device through metallic electrodes e + and e- for a fast discharge. S1 and S2 are superconducting material layers. There are very thin insulated layers between $\mathrm{S} 1$ and $\mathrm{e}+$ as well as between S2 and e-. There is a partially conducting contact between S1 and S2. The device is immersed in liquid nitrogen not shown here. $\Phi$ is the vertical axis of the device.

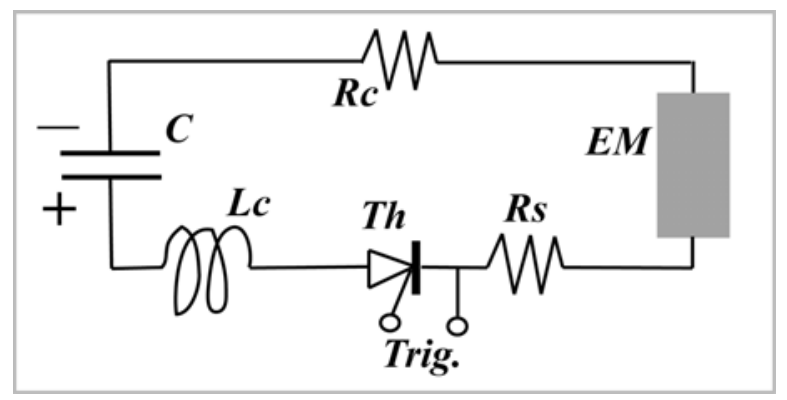

Figure 2. Circuit generating the discharge

Electric energy of the discharges is stored into capacitor C. The circuit has a distributed inductance Lc and a distributed resistance Rc. The discharges are sent into the emitter EM through the remotely-controlled thyristor Th. A fixed resistor Rs $(0.13 \pm 0.005 \Omega)$ is added to avoid oscillation of the discharge current which is measured by using Rs as a shunt. 


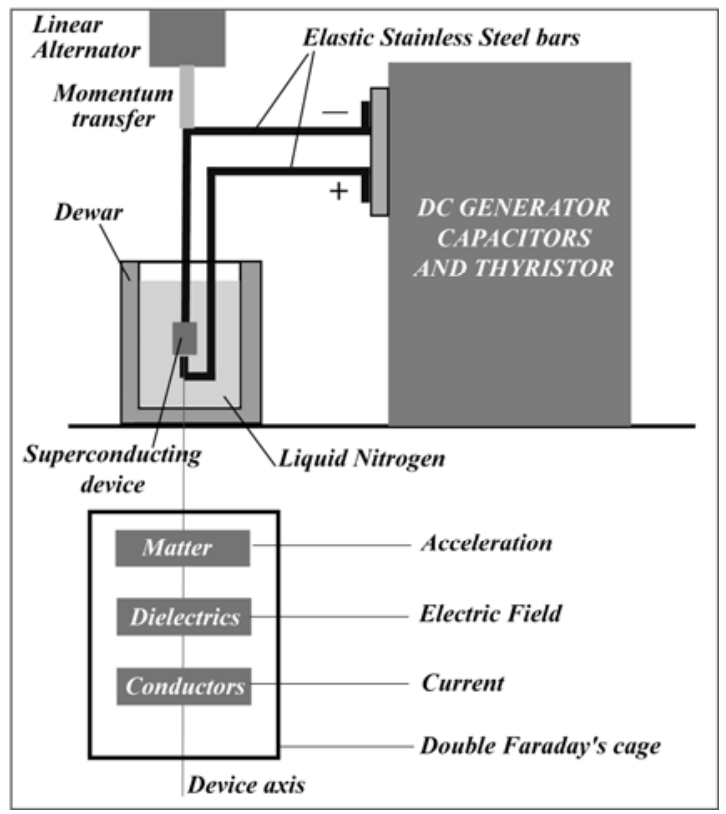

Figure 3. Experimental system

It uses four parts: - An emitter support with elastic stainless steel bars conducting the discharge current and holding the emitter in liquid nitrogen. - A discharge system, with a high voltage power supply, energy storage capacitors, thyristor switch, cryostat Dewar, and boiling liquid nitrogen. - A linear alternator which mobile magnets were pushed by an insulated momentum transfer rod. - Measuring devices enclosed in an independent double Faraday's cage, under the cryostat, along the vertical emitter axis. Eight sensors measured essentially the vertical acceleration of matter, the electric field induced inside dielectrics, and the current induced inside longitudinal conductors.

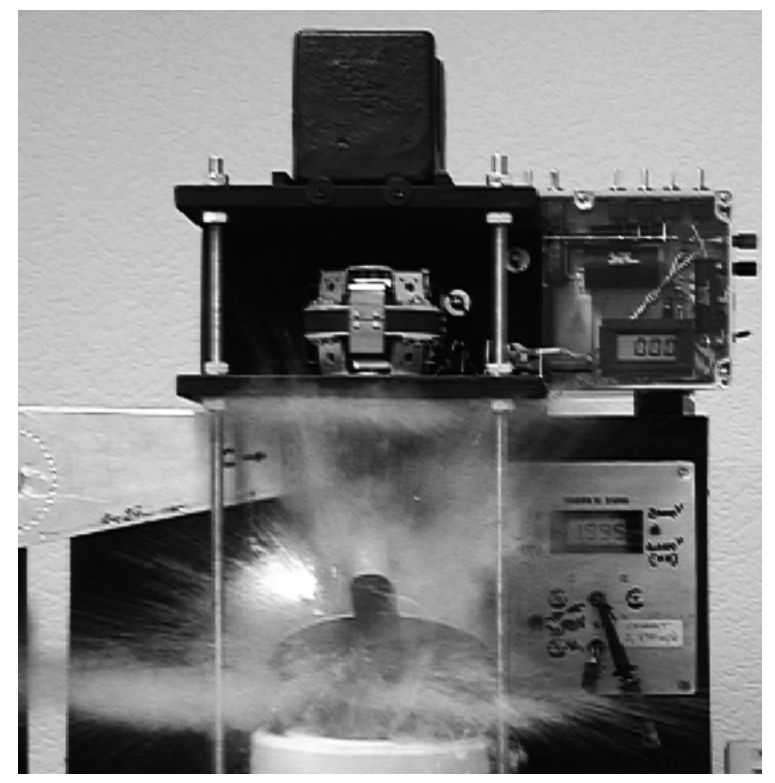

Figure 4. Propelling phenomenon, image from an experimental video movie

This still image corresponds to the beginning of a 1995 volts discharge into a pair of emitters connected in series (EM118+119). We propose a 57 seconds QuickTime movie with sound showing seven discharges of different voltages including one into a false emitter made of copper plates immersed in liquid nitrogen. The whiplash-like sound emitted is heard during each of the six discharges made into layered emitters and waves from the distant water tank are briefly observed. In this movie, the propelling momentum from the emitter is partially transferred with a delay to the linear alternator by a horizontal pendulum, for ease of observation (available online). 


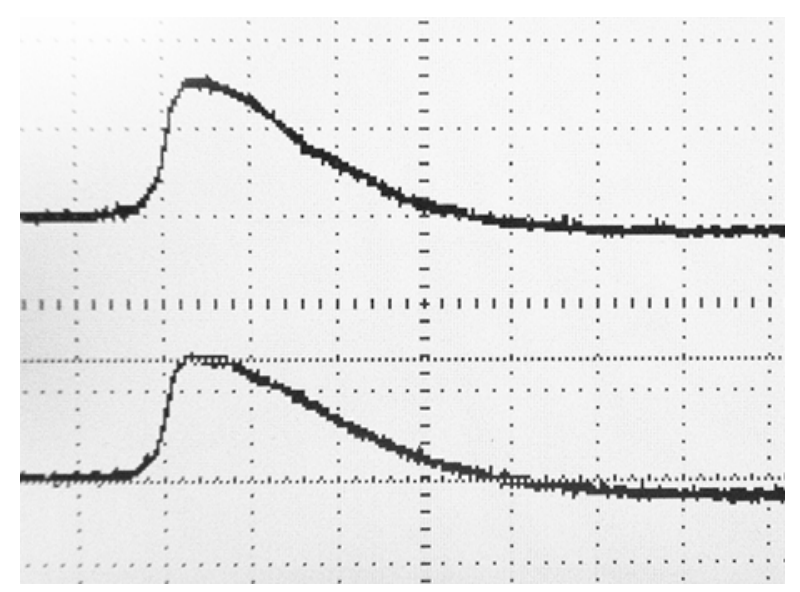

Figure 5. Emitter voltage (upper curve) and current

These were recorded during a $376 \pm 1 \mathrm{~V}$ discharge in emitter EM3 cooled down to liquid nitrogen temperature. Peak voltage is $105 \pm 2 \mathrm{~V}$, peak current is $860 \pm 5 \mathrm{~A}$. Peak power is $90 \mathrm{~kW}$. Scales: $66 \pm 1 \mathrm{~V} / \mathrm{div}, 614 \pm 5 \mathrm{~A} /$ $\operatorname{div}, 10 \pm 0.01 \mu \mathrm{s} / \operatorname{div}$.

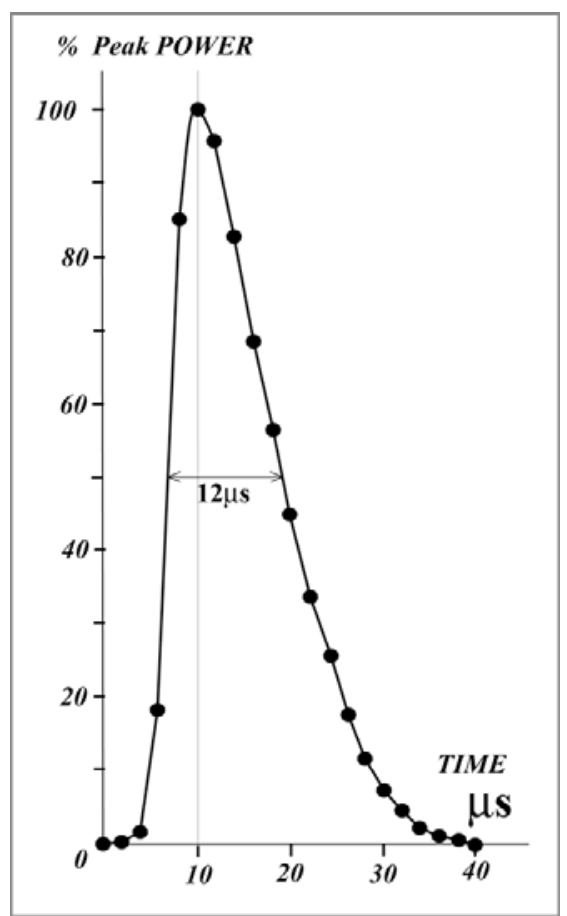

Figure 6. Electrical power versus time

Power applied to the emitter during the discharge shown in Fig. 5. 


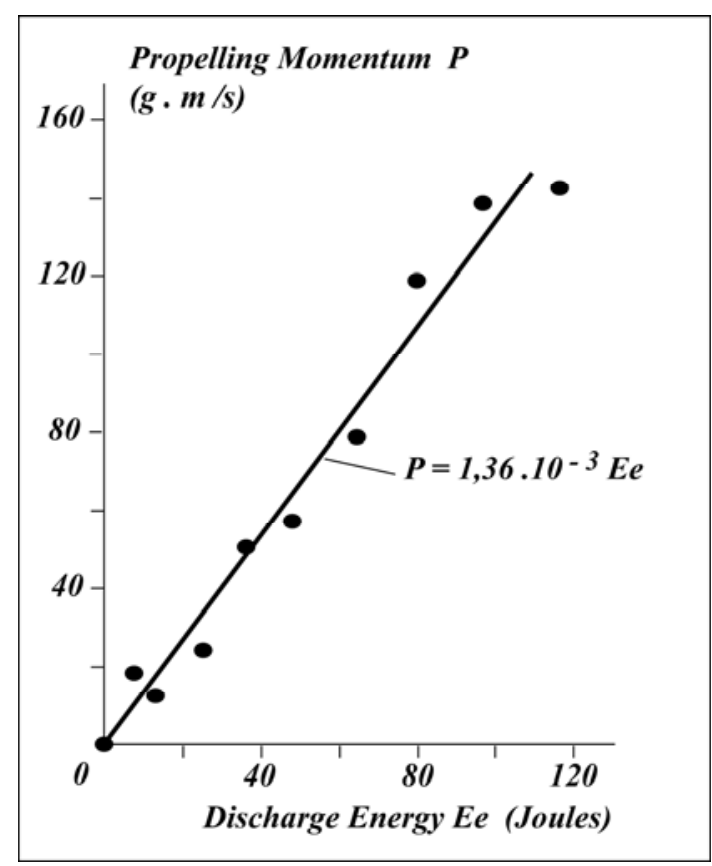

Figure 7. Typical example of propulsive momentum versus discharge energy

Momentum is proportional to electric energy of the discharge. The dot size corresponds to the measurements error (one $\sigma)$.

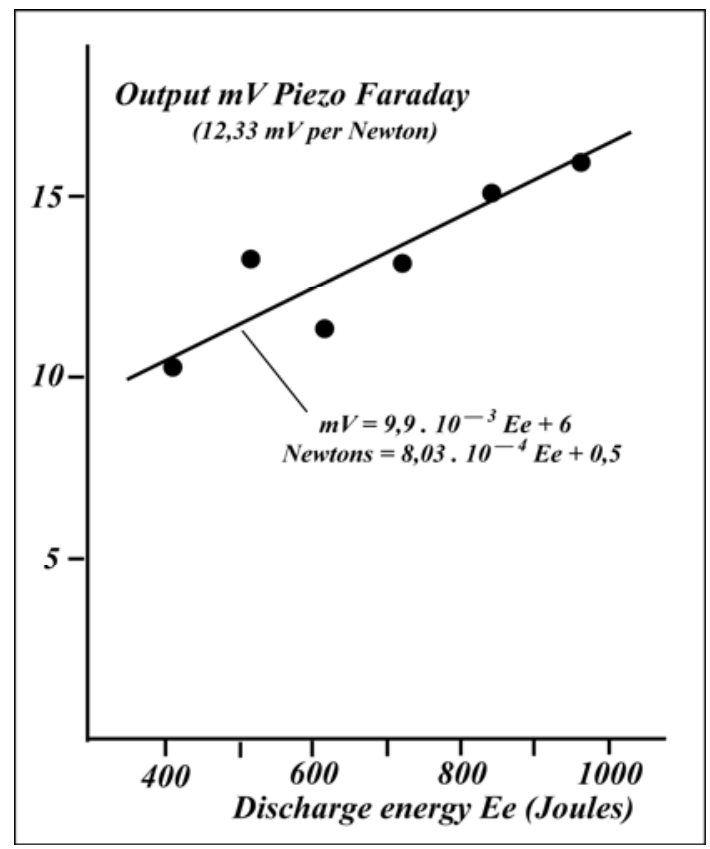

Figure 8. Typical output voltage of the piezoelectric accelerometer versus discharge energy into an emitter The output signal is proportional to the electric energy of the discharge. The peak to peak noise was about $1 \mathrm{mV}$. This kind of accelerometer shows a bias DC voltage at rest because of the gravity acceleration. 


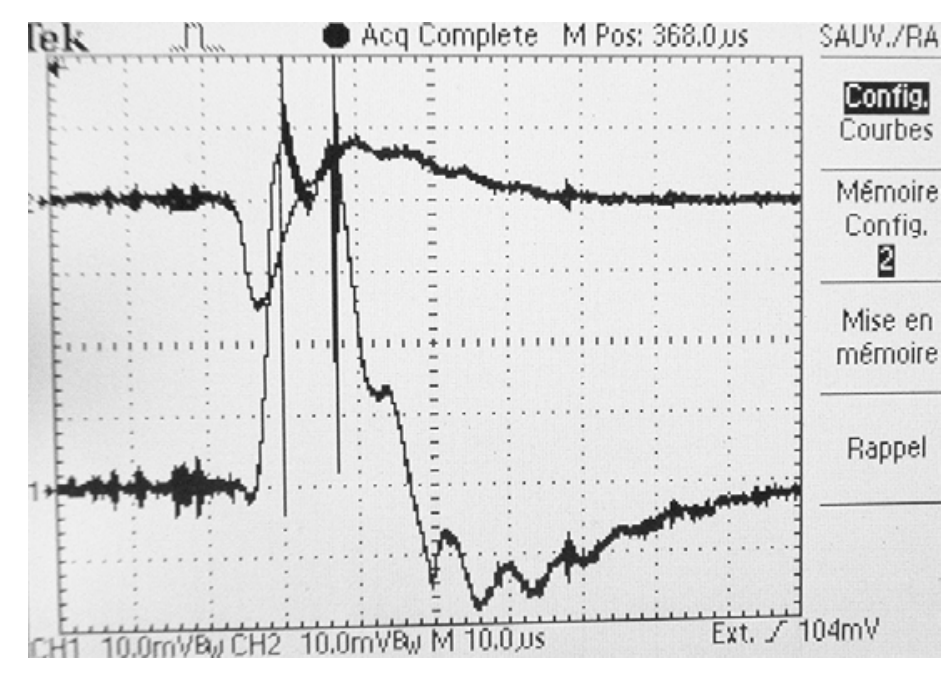

Figure 9. Output signals from two detectors separated by a distance of $95 \pm 0.1 \mathrm{~cm}$, recorded during a discharge in EM8 emitter

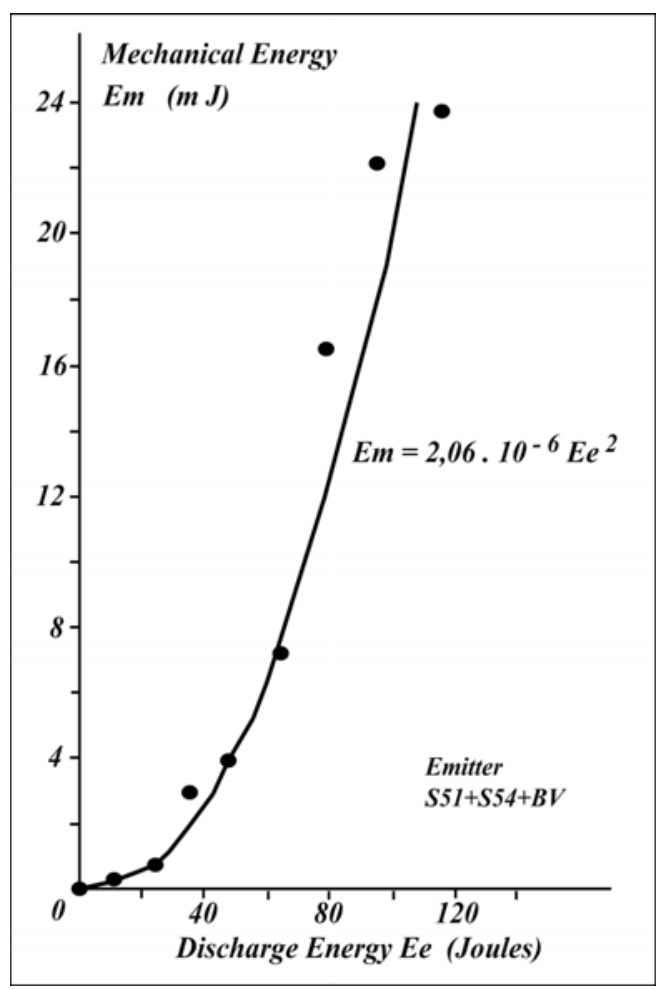

Figure 10. Kinetic energy transferred to the mobile magnets of the linear alternator, versus the electric energy into the bi-layered emitter S51+S54+BV

The evolution of output mechanical energy versus discharge electric energy is in agreement with expression (3). 